\title{
Dynamic Address Routing for Adhoc \& Mesh Networks using MANETs
}

\author{
Indira Priyadarshini Tummala ${ }^{1, *}$, Ramya Sree $\mathrm{B}^{1}$ \\ ${ }^{1}$ CSE Department, Gokaraju Rangaraju Institute of Engineering \& Technology, Hyderabad, INDIA
}

\begin{abstract}
The organization ought to have the option to adaptively change the directing ways to lighten any of these impacts. In addition, in a tactical climate, protection of safety, inertness, dependability, deliberate sticking, and recuperation from disappointment are huge concerns. Military organizations are intended to keep a low likelihood of catch and additionally a low likelihood of recognition. Henceforth, hubs like to emanate as little force as essential and send as inconsistently as could be expected, accordingly diminishing the likelihood of discovery or interference. A pass in any of these prerequisites may corrupt the exhibition and constancy of the organization. The fundamental target of this product is to make a way for exchange utilizing dynamic locations in a look into table. This look-into table is alluded and kept up during correspondence by every one of the hubs in the organization. The look-into table is refreshed on each occurrence regardless of whether there is any hub nonattendance. The situation with every hub in the organization is additionally kept up in the all the look-into tables.
\end{abstract}

Keywords: MANETs, Networks, Adhoc Networks, Scalable.

\section{Introduction}

An Ad-hoc network is a bunch of remote portable hubs that structure a powerful self-governing organization without the intercession of unified passageways or base stations. In contrast to customary remote organizations, Ad-hoc networks require no fixed organization foundation [1] and can be conveyed as multi-bounce parcel networks quickly and with generally low cost. Instances of utilizations incorporate front lines, crisis administrations, meeting rooms, and home and office [2]. Versatile hubs in an Ad-hoc network have restricted radio transmission range. Hubs that cannot discuss straight forwardly with one another necessitate that moderate hub forward bundles for them. Every hub act both as a switch and as a host.

The capacity of a steering convention in Ad-hoc network is to set up courses between various hubs. Weaknesses, and can be assaulted. The unique attributes of Ad-hoc networks set forward difficulties do not present in conventional wired organizations. In the customary Internet, switches inside the focal pieces of the organization are possessed by a couple of notable administrators and are hence thought to be trust worthier [3]. This presumption no longer holds in an Ad-hoc network since neither halfway administrated secure switches nor a severe security strategy exists in an Adhoc organization, and all hubs entering the organization are relied upon to partake in directing [4]. Moreover, because the geography in such an organization can be profoundly unique, customary steering conventions can at this point do not be utilized. Hence, an Ad-hoc network has a lot higher security prerequisites than the customary organizations and the steering in Ad-hoc networks is difficult to achieve safely, heartily, and effectively. The universally useful of getting Ad-hoc steering conventions is to secure the directing messages, to keep aggressors from changing these messages or in any event, infusing destructive directing messages into the organization. Thus, honesty and validness of directing messages ought to be ensured. Privacy can be guaranteed effectively, e.g., by encryption, yet it will build overhead [5].

In the best-in-class time of distant correspondence structures, there will be a necessity for the quick association of independent flexible customers. Immense models consolidate setting up survivable, useful, amazing correspondence for emergency/rescue exercises, disaster help adventures, and military associations [6-7]. Such association circumstances can't rely upon joined and composed accessibility and can be envisioned as employments of Mobile Ad Hoc Networks. A MANET is an independent combination of convenient customers that pass on over by and large information transmission obliged far off associations. Since the center points are adaptable, the association topography might change rapidly and curiously as time goes on [8]. The organization is decentralized, where all organization action including finding the geography and conveying messages should be executed by the actual

\footnotetext{
* Corresponding author: priya230@gmail.com
} 
hubs, i.e., steering usefulness will be fused into portable hubs.

The plan of employments for MANETs is unique, going from close to nothing, static associations that are obliged by power sources, to colossal extension, convenient, significantly remarkable associations. The arrangement of association shows for these associations is a stunning issue [9]. In any case the application, MANETs need useful scattered estimations to choose network affiliation, associate arranging, and coordinating. In any case, choosing viable coordinating ways and passing on messages in a decentralized environment where network topography wavers is certifiably not a particular issue. While the most restricted way (considering a given cost work) from a source to a target in a static association is typically the best course, this thinking isn't helpfully loosened up to MANETs. Elements like variable far off association quality, causing way incident, obscuring, multi-customer obstacle, impact utilized, and topological changes, become critical issues [10]. The association should have the choice to adaptively change the guiding approaches to ease up any of these effects. Additionally, in a strategic environment, defending of wellbeing, dormancy, steadfastness, intentional staying, and recovery from frustration are tremendous concerns. Military associations are planned to keep a low probability of catch just as a low probability of recognizable proof [11]. Subsequently, hubs like to emanate as little force as vital and communicate as inconsistently as could really be expected, in this manner diminishing the likelihood of recognition or capture attempt. A pass in any of these necessities may corrupt the exhibition and reliability of the organization. The fundamental goal of this product is to make a way for exchange utilizing dynamic locations in a look into table [12]. This look-into table is alluded and kept up during correspondence by every one of the hubs in the organization. The look-into table is refreshed on each occurrence regardless of whether there is any hub nonappearance. The situation with every hub in the organization is additionally kept up in the all the lookinto tables.

\section{Related Work}

Assume that we need to associate two office floors utilizing short-range remote specialized gadgets effectively and proficiently. Each worker has one of these cell phones, and some fixed gadgets PCs, printers, etc; have a similar capacity [13]. We could interface these gadgets to the current wired foundation utilizing passageways, however this choice offers restricted, portability, includes load the wired organization, and depends on existing conventions for wired correspondence. Another chance is to fabricate an organization of committed and physically associated base stations that empower cell correspondence, yet this is costly as for time, establishment, and support.

The best arrangement is to make a versatile impromptu organization utilizing encompassing electronic gadgets as middle of the road switches when they are inactive and if they can play out this assignment. For instance, the parcel from one gadget can bounce to the cell phone of an individual going through the passageway before the workplace, then, at that point from the cell phone to the common laser printer in the following office, then, at that point to somebody's computerized wristwatch on the floor beneath, then, at that point from the wristwatch to the espresso machine, and, at last from the espresso machine to its definitive objective say another associate's gadget or PC [14].

MANETs are additionally helpful for calamity the board. An interchanges foundation is intended to endure normal momentary issues, for example, over-burdening, however, not to support major actual harm. Much of the time, the breakdown of a solitary framework will make numerous reliant gadgets fall flat. On the off chance that a fire, quake, or other normal disaster debilitates a subset of base stations, each cell phone inside scope of those stations naturally gets inaccessible. In such circumstances, salvage laborers can utilize the hubs in MANETs to make an organization "on the fly". Limited scope MANETs are likewise successful for crisis search and salvage, front line observation, [15] and other correspondence applications in perilous conditions. For instance, robots or self-governing sensors conveyed in a space blocked off to people could utilize straightforward magnet steering conventions to communicate information to a control place. Regardless of whether numerous robots or sensors are debilitated or obliterated, the excess ones would have the option to reconfigure and keep sending data. Dynamic addressing is a plausible method to accomplish versatile specially appointed directing. "Versatile" signifies thousands up to a great many hubs in a specially appointed or network organization. It isolates the directing location and the character of a hub. The steering address of a hub is dynamic and changes with hub development to mirror the hub's area in the organization geography [5]. The identifier is an all around the world novel number that remains something very similar all through the lifetime of the hub.

Versatility is a necessity in systems administration innovations to arrive at their maximum capacity. Specially appointed systems administration innovation has progressed hugely during the most recent ten years, yet it still cannot seem to turn into a broadly sent innovation. The current directing conventions and structures function admirably simply up to a couple hundred hubs [10]. Most ebb and flow research in specially appointed organizations centre more around execution and force utilization related issues in moderately little organizations, and less on adaptability. The fundamental explanation for the absence of versatility is that these conventions depend on level and static tending to [6]. With versatility as a fractional objective, a few endeavours have been made toward various levelled steering and grouping. These methodologies do hold guarantee, yet they do not appear to be effectively sought after. 


\section{System Analysis}

\subsection{Definition}

The organization design, which could use the new tending to plot adequately. Truth be told, dynamic steering and tending to shape the reason for a novel systems administration layer, which we portray in some detail in our prior work. In this methodology, we separate the steering address and the character of a hub. The steering address of a hub is dynamic and changes with hub development to reflect the hub's area in the organization geography. The identifier is an around the world one of a kind number that remains something similar all through the lifetime of the hub. For simplicity of show, we can expect for the time being that every hub has a solitary identifier.

We recognize three significant capacities. To start with, address portion keeps one directing location for each organization interface, so that the location demonstrates the hub's relative organization area. Second, steering conveys parcels from a hub to a given directing location. Third, hub query is a conveyed query table planning each hub identifier to its present organization address. Let us first portray how we need things to function according to a functional perspective. At the point when a hub joins the organization, it pays attention to the intermittent directing updates of its adjoining hubs and utilizations these to distinguish an abandoned location. We will depict how this is done later. The joining hub enrolls its special identifier and the recently gotten address in the appropriated hub query table. Because of versatility, the location may in this manner be changed and afterward the query table should be refreshed. At the point when a hub needs to send bundles to a hub known simply by its identifier, it will utilize the query table to find its present location [9].

We start by introducing two perspectives on the organization that we use to portray our methodology: a) the location tree, and b) the organization geography.

Note that the arrangement of hubs from any sub tree initiates an associated sub diagram in the organization geography. This isn't a fortuitous event, however a critical property of our dynamic tending to approach. Naturally, hubs that are near one another in the location space ought to be moderately close in the organization geography. More officially, we can express the accompanying constraint.1) Prefix Sub chart Constraint: The arrangement of hubs that share a given location prefix structure an associated sub diagram in the organization geography. This requirement is central to the adaptability of our methodology. Instinctively, this imperative assists us with planning the virtual pecking order of the location space onto the organization geography. The more extended the common location prefix between two hubs, the more limited the normal distance in the organization geography.

At last, let us define two new terms that will work with the conversation in the accompanying segments. A Level-sub tree of the location tree is defined by a location prefix of pieces, as displayed in Fig. 1. For instance, a Level-0 sub tree is a solitary location or one leaf hub in the location tree. Bit prefix and can contain up to A Level-1 sub tree has two leaf hubs. In Fig. 1, [0xx] is a Level-2 sub tree containing addresses [000] through [011]. Note that each Level-sub tree. We define the term Level-kin of an offered address to be the sibling3 of the Level-sub tree to which a given location has a place. By drawing whole kin sub trees as triangles, we can make disconnected perspectives on the location tree.

\subsection{Proposed System}

Dynamic addressing is a doable method to accomplish versatile specially appointed directing. "Adaptable" signifies thousands up to a great many hubs in a specially appointed or network organization.

$>$ It isolates hub personality from hub address and uses the location to show the hub's present area in the organization.

$>$ Address designation conspire utilizes the location space proficiently on geographies of arbitrarily and consistently dispersed hubs, exactly bringing about normal directing.

$>$ In presently use IP address as identifier, which is an internationally novel number that remains something very similar all through the lifetime of the hub. In this way, the vehicle and application layers don't have to change, and the steering address is just seen at the organization layer.

It insatiably limits the normal size of the subsequent directing table at every hub. Nonetheless, it might need to redistribute addresses proactively to improve: 1 ) the adjusting of the location tree, and 2) the length of the directed ways.

\section{Architecture Design}

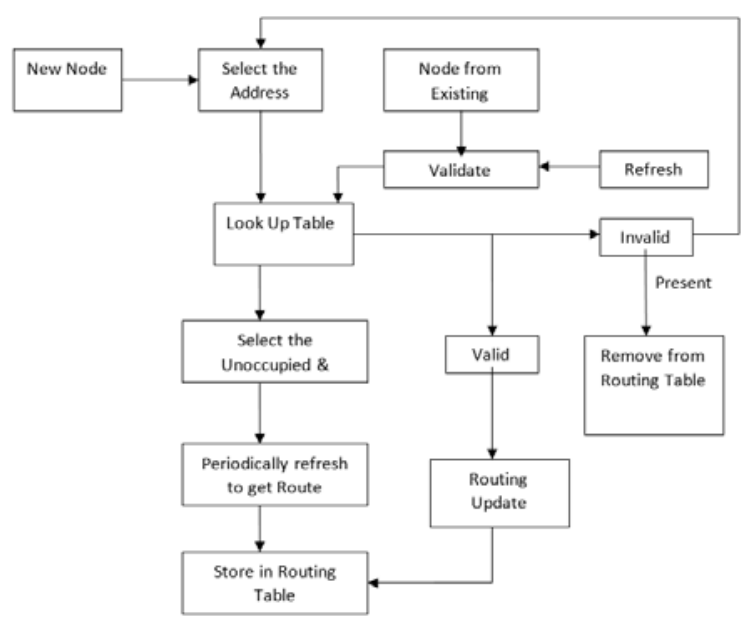

Fig. 1. Architecture Diagram.

Framework configuration is worried about how the framework practically is to be given by the various parts of the framework. The framework configuration measure 
partion the necessities to one or the other equipment or programming framework. It builds up framework engineering. Programming configuration includes recognizing and portraying.

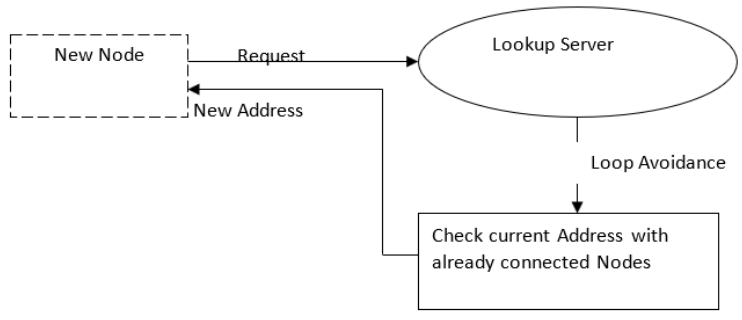

Fig. 2. Routing State of Node with Loop Avoidance Module 1

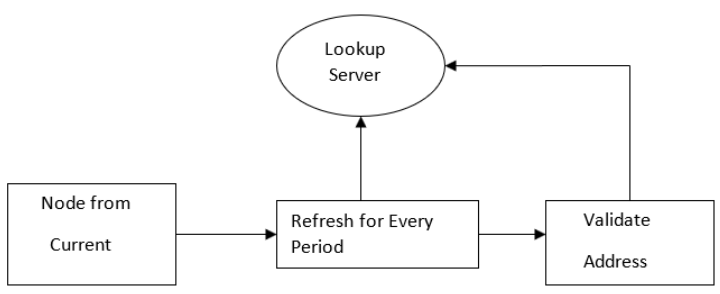

Fig. 3. Routing State of Node from current

\section{IMPLEMENTATION}

\subsection{Modules}

For the ease of coding, our project is divided into four modules. They are as follows:

1. Routing State of Node with Loop Avoidance

2. Node Lookup

3. Dynamic Address Allocation

$>$ Address Selection with Best Neighbour

$>$ Address Validation

4. Populating and Maintaining the Routing Table

$>\quad$ Refresh and Routing Update Structure

\subsubsection{Routing state of node with loop avoidance}

Every hub keeps some directing state, as determined with regards to a kin hub level which is put away at position. Naturally, the directing state for kin contains the data important to keep a course toward a hub (any hub) in that sub tree. DART utilizes the organized location space to make another sort of circle evasion plot. To save versatility, this paper sums up the circle opportunity rule. For each sub tree, when a steering passage has left the sub tree, it isn't permitted to return. This adequately forestalls circles and can be executed in an exceptionally adaptable way.

\subsubsection{Node lookup}

Track down the missing connection of the current location of a hub. This paper proposes to utilize a circulated hub query table, which maps every identifier to a location. Hub sections are characterized in Anchor Node of the identifier. This paper picked a technique for intermittent revive, where each hub occasionally sends its data to moor hub. It sets all query table sections to terminate consequently after a period twice if the occasional invigorate span.

\subsection{Dynamic Address Allocation}

At the point when a hub joins a current organization, it utilizes the occasional steering updates of its neighbours to distinguish and choose an abandoned and genuine location. It begins by choosing which neighbour to get a location from the neighbour with the most elevated level addition point is chosen as the best neighbour. The addition point is characterized as the most significant level for which no directing section exists in each neighbour's steering table. For each neighbour, track down the most noteworthy request bit where the current hub's location varies from the neighbour's location. Assuming the addresses are indistinguishable, the location is invalid if the neighbour has a lower identifier. Something else, check the neighbour's declared directing table for the passage that ought to contain our sub tree. Assuming a passage exists, however contains an alternate identifier, we have distinguished an addressing struggle that should be settled. The current location is invalid if the privately figured identifier is bigger.

\subsection{Module Explanation}

DART utilizes a clever plan for distinguishing and trying not to defeat circles, which use the various leveled nature of the location space to further develop adaptability. To start with, let us audit the overall idea of circle evasion, to establish the framework for the conversation of our circle aversion plot. From a theoretical perspective, directing circle evasion is tied in with recollecting what hubs a course update has navigated and ensuring that these hubs don't acknowledge course refreshes that they have effectively seen. On the off chance that this necessity is satisfied, directing circles can't happen.

A basic method of carrying out this is to connect a rundown of all visited hubs in the directing update, and to have hubs really look at this rundown prior to tolerating an update. Nonetheless, this methodology has an adaptability issue, in that steering updates will rapidly develop too inconvenient sizes. All things considered; Dynamic Address Routing utilizes the organized location space to make another sort of circle aversion conspire. To save versatility, we sum up the circle opportunity rule above. For each sub tree, when a steering passage has left the sub tree, it isn't permitted to return. This successfully forestalls circles and can be carried out in an exceptionally versatile way: A bit cluster of ADDR SIZE pieces are kept along with the steering update. Bit of the course log demonstrates whether the current hub through the level-kin course update showed up at the current hub by means of the level-kin. 


\subsection{Dynamic Address Allocation}

To survey the attainability of dynamic tending to, we foster a set-up of conventions that execute such a methodology. Our work adequately takes care of the principle algorithmic issues and structures a steady system for additional dynamic tending to explore. Albeit the plan has not yet been enhanced for most extreme throughput, its adaptability properties and unsurprising execution show guarantee.

\subsection{Address Validation}

The legitimacy of a location is confirmed by checking it against the steering tables, all things considered. For each neighbour, track down the most elevated request bit where the current hub's location contrasts from the neighbour's location. Assuming the addresses are indistinguishable, the location is invalid if the neighbour has a lower identifier. Something else, check the neighbour's reported directing table for the passage that ought to contain our sub tree. Assuming a section exists, yet contains an alternate identifier, we have distinguished an addressing struggle that should be settled. The current location is invalid if the privately processed identifier is bigger.

\subsection{Refresh and Routing Update Structure}

Each period, every hub executes Refresh. It really looks at the legitimacy of its present location, populates a directing table utilizing the data got from its neighbors, and broadcasts a steering update.

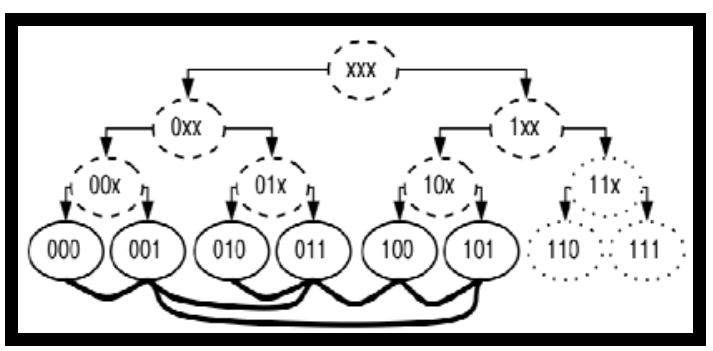

Fig.4. Lookup Table Representation

Table 1 Lookup Table

\begin{tabular}{|c|c|}
\hline Level & Next Hop \\
\hline 0-Level (010) & 010 \\
\hline 1-Level (00X) & 001 \\
\hline 2-Level (1XX) & 100 \\
\hline
\end{tabular}

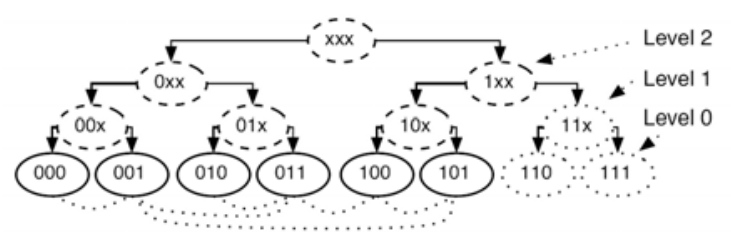

Fig.5. Routing Table Representation

\section{Testing \& Discussions}

Testing is a bunch of exercises that can be arranged and led efficiently. Thus, a format for programming testing-a bunch of steps into which we can put explicit experiment plan strategies and testing techniques ought to be characterized for programming measure. In unit testing the modules of the framework are tried as individual units. Every unit has clear information and yield boundaries and frequently a positive single capacity. Thus, unit testing is also called program testing. A program unit is typically little sufficient that the developer who created can test in a detail and surely more meticulously that will be conceivable when the unit is coordinated into an advancing programming item. There are four classes of tests that a software engineer will commonly perform on a program unit. In framework testing, the framework is tried as entire; that is intercommunication among the singular units and elements of the total framework are tried. Incorporation testing is a methodical testing for building the program structure while simultaneously directing test to uncover blunders related with the interfacing. While performing mix testing, every one of the modules of the undertaking were incorporated all in all tasks and checked whether the right yield was created. The goal is to take unit-tried parts and assembled a program structure that has been directed by the plan.

\subsection{Validation Testing}

Programming approval is accomplished through a progression of discovery test that show similarity with necessities. Test plan diagrams classes of test to be led and test strategy characterizes the experiments that will be utilized to exhibit similarity with prerequisites. Both the arrangement and system are intended to guarantee that all useful prerequisites are fulfilled, all social attributes are accomplished, and all exhibition necessities are achieved.

\subsection{Maintenance}

Once the project or system is subjected to various testing techniques, the final stage of software development lifecycle is maintenance. After the product is being installed at the customer end, we carry out maintenance by continuously monitoring the performance of the system. When there is a reduction in the level of efficiency, the cause and effects are analysed and rectified.

\section{Conclusion}

Dynamic addressing is a practical method to accomplish adaptable impromptu directing. "Versatile" signifies thousands up to many hubs in an impromptu or cross section organization. It isolates hub character from hub address and uses the location to show the hub's present 
area in the organization. Address allotment conspire utilizes the location space effectively on geographies of arbitrarily and consistently appropriated hubs, exactly bringing about normal steering. In at present use IP address as identifier, which is an all around the world exceptional number that remains something similar all through the lifetime of the hub. Subsequently, the vehicle and application layers don't have to change, and the directing location is just seen at the organization layer. It ravenously limits the normal size of the subsequent directing table at every hub. Notwithstanding, it might need to redistribute addresses proactively to improve: 1) the adjusting of the location tree, and 2) the length of the directed ways

\section{References}

1. Jakob Eriksson, Michalis Faloutsos, and Srikanth Krishnamurthy, Scalable ad hoc routing: The case for dynamic addressing, IEEE InfoCom, (2004).

2. Kavitha Merugu, Prasad Dinkar, Bobba Phaneendra Babu, IET Electric Power Appl., 13, 8 (2019).

3. B. Phani Teja, V. Srikanth Babu, T. Suresh Kumar, Int. Jol. of App. Engg. Research 10, 16 (2015).

4. Brett Warneke, Matt Last, Brian Liebowitz, and Kristofer S. J. Pister, Computer, vol. 34, 1 (2001).

5. Ram Ramanathan and Martha Steenstrup, Mobile Networks and Applications, 3, 1 (1998).

6. M. Kavitha, P. B. Bobba and D. Prasad, Investigations and experimental study on Magnetic
Resonant coupling based Wireless Power Transfer system for neighborhood EV's, (IEEE ICPS 2016).

7. Guangyu Pei, Mario Gerla, Xiaoyan Hong, and Ching-Chuan Chiang, $A$ wireless hierarchical routing protocol with group mobility, WCNC, 1999.

8. G. Pei, M. Gerla, and X. Hong, Lanmar: Landmark routing for large scale wireless ad hoc networks with group mobility, ACM MobiHOC'00, 2000.

9. X. Hong, M. Gerla, G. Pei, and C. Chiang, A group mobility model for ad hoc wireless networks, 1999.

10. J. Eriksson, M. Faloutsos, and S. Krishnamurthy, Peernet: Pushing peer-2-peer down the stack., IPTPS, 2003.

11. B. J. Varghese, P. B. Bobba and M. Kavitha, Effects of coil misalignment in a four-coil implantable wireless power transfer system, (IEEE PIICON 2016).

12. C. Perkins, ad hoc on demand distance vector routing, 1997.

13. Charles Perkins and Pravin Bhagwat, highly dynamic destination sequenced distance-vector routing for mobile computers, ACM SIGCOMM, 1994.

14. David B Johnson and David A Maltz, Mobile Computing, 35, 3(1996).

15. S. Basagni, I. Chlamtac, V. R. Syrotiuk, and B. A. Woodward, A distance routing effect algorithm for mobility, ACM/IEEE MobiCom, 1998 